Review

\title{
Body of evidence supporting the clinical use of 3D multisegment foot models: A systematic review
}

\author{
Kevin Deschamps ${ }^{\mathrm{a}, \mathrm{b}, \mathrm{c}, \mathrm{d}, *}$, Filip Staes ${ }^{\mathrm{b}}$, Philip Roosen ${ }^{\mathrm{e}}$, Frank Nobels ${ }^{\mathrm{f}}$, Kaat Desloovere ${ }^{\mathrm{c}, \mathrm{g}}$, \\ Herman Bruyninckx ${ }^{\text {h }}$, Giovanni A. Matricali ${ }^{\text {a }}$ \\ a Division of Musculoskeletal Disorders, Multidisciplinary Diabetic Foot Clinic, University Hospitals Leuven, Katholieke Universiteit Leuven, Weligerveld 1, \\ 3212 Pellenberg, Belgium \\ ${ }^{\mathrm{b}}$ Department of Rehabilitation Sciences-Research Center for Musculoskeletal Rehabilitation, Katholieke Universiteit Leuven, Tervuursevest 101, \\ B-3001 Leuven (Heverlee), Belgium \\ ${ }^{\mathrm{c}}$ Laboratory for Clinical Motion Analysis, University Hospital Pellenberg, KU-Leuven, Belgium

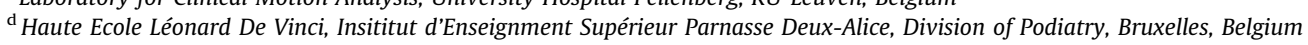 \\ ${ }^{\mathrm{e}}$ Department of Rehabilitation Sciences and Physiotherapy, Research Group: Musculoskeletal Rehabilitation, University Gent, Campus Heymans (UZ Gent), \\ Blok B3, De Pintelaan 185, 9000 Gent, Belgium \\ ${ }^{\mathrm{f}}$ Department of Internal Medicine-Endocrinology, Multidisciplinary Diabetic Foot Clinic, Onze-Lieve-Vrouw Ziekenhuis Aalst, Moorselbaan 164, 9300 Aalst, Belgium \\ ${ }^{\mathrm{g}}$ Department of Rehabilitation Sciences, Katholieke Universiteit Leuven, Weligerveld 1, 3212 Pellenberg, Belgium \\ ${ }^{\mathrm{h}}$ Department of Mechanical Engineering, Katholieke Universiteit Leuven, Celestijnenlaan 300B Bus 2420, Room 01.053, B-3001 Leuven (Heverlee), Belgium
}

\section{A R T I C L E I N F O}

\section{Article history:}

Received 5 August 2010

Received in revised form 13 December 2010

Accepted 16 December 2010

\section{Keywords:}

Foot

Kinematics

3D multisegment foot model

Motion

\begin{abstract}
A B S T R A C T
Background: A critical component in the characterization of foot mechanics during clinical gait analysis is the quantitative measurement of foot kinematics. Currently, the use of 3D multisegment foot models (3DMFMs) is popular in gait laboratories as it would seem to be an adequate tool for the in vivo analysis of dynamic foot kinematics. This systematic review identifies and evaluates current evidence for the use of 3DMFMs in clinical gait analysis.

Methods: A targeted search strategy traced full papers that fulfilled the inclusion and exclusion criteria. The papers were classified and evaluated for quality using a custom made quality appraisal form. Findings: Forty-one manuscripts were included yielding a total number of fifteen 3DMFMs. Generally, study procedures and sample selection were adequately described; however, the methodological quality varied widely. Evidence regarding the repeatability of the identified models also varied widely. Models facing the highest level of scientific credibility were characterized by adequate repeatability indices obtained from between-trial, between-day and between and within assessor studies. Generally, the highest reliability indices were found for the sagittal plane kinematics. Within-subject variability was found to be the lowest, contrarily, between-subject and between-day variabilities were found to be highest.

Interpretation: Reported repeatability indices such as the coefficient of multiple correlation, standard deviation and standard error of measurement provide evidence for the continued use of 3DMFMs. While a number of published models exist, there is no adequate evidence available to support their clinical use. More reliability and validity studies are needed to confirm adequate measurement properties of 3DMFMs. (c) 2010 Elsevier B.V. All rights reserved.
\end{abstract}

\section{Introduction}

The analysis of foot mechanics during walking or other activities has been a major challenge for many years. Qualitative interpretations with only moderate scientific evidence are most often used for current concepts and paradigms of foot function. The inability to measure in vivo foot kinematics has been a particular problem [1,2].

\footnotetext{
* Corresponding author at: Division of Musculoskeletal Disorders, University Hospital-Leuven, KU-Leuven at Weligerveld 1, 3212 Pellenberg, Belgium. Tel.: +32 16338024 .

E-mail address: kevin.deschamps@uz.kuleuven.ac.be (K. Deschamps).
}

Three-dimensional (3D) stereophotogrammetric analysis is the state of the art method for the analysis of joint kinematics in humans. Two approaches are generally applied. One approach is the use of intracortical bone pins with makers on top and can be considered to be the 'gold standard' as it represents the actual motion of the bones [3,4]. However, the invasive nature limits the clinical use. Another approach uses skin-mounted markers, arrays of markers or a combination of both and is the most frequently used in gait laboratories [5]. During the last decade, a number of research groups have used the latter approach to model the foot according to a number of small segments, generally referred to as 3D multisegment foot models (3DMFMs). The fact that these published 3DMFMs share some fundamental aspects (e.g. marker 
Table 1

Inclusion and exclusion criteria considered for the current systematic review.

\begin{tabular}{|c|c|}
\hline Criteria & Applied definition \\
\hline Multisegment approach & $\begin{array}{l}\text { An approach using at least three segments (excluding the tibia). Segments are determined based on the strategic placement of a } \\
\text { number of markers and is typically performed in a systematic and rationalized way in order to gain insight in the kinematic } \\
\text { behaviour of the foot. }\end{array}$ \\
\hline $\begin{array}{l}\text { 3D motion analysis of } \\
\text { the foot }\end{array}$ & $\begin{array}{l}\text { Collecting kinematic data by recording the motion of markers affixed to a moving person using a motion-caption system. Three- } \\
\text { dimensional coordinates of each marker, obtained by a digitizing procedure, is subsequently used as basis for the calculation of } \\
\text { segmental or joint angles. }\end{array}$ \\
\hline $\begin{array}{l}\text { In-vivo/non-invasive } \\
\text { motion analysis }\end{array}$ & $\begin{array}{l}\text { Kinematic analysis performed on living subjects using surface markers. The markers are directly applied on the skin of the subject } \\
\text { or mounted on top of a rigid plate (clusters). Studies including cadavers or bone pins (invasive) do not fulfil this criterion. }\end{array}$ \\
\hline Weight bearing analysis & $\begin{array}{l}\text { Testing and evaluation of the foot during weight bearing activities such as standing, walking, jumping, descent walking, and } \\
\text { jogging. Focus of these analyses is the weight bearing function of the foot. }\end{array}$ \\
\hline Cohorts under investigation & No restrictions were applied regarding the sex or age of the participants. \\
\hline
\end{tabular}

placement, joint angle calculation, and segment definition) has led to the general consensus about the appropriateness of representing the foot as a small number of segments [6,7]. Although this technique has some weaknesses related to skin motion artifact and the difficulty of defining specific bones in the foot [8-11], it has the potential to overcome the weaknesses of the single-segment foot models, which are often used in clinical gait analysis, associated with conventional lower limb models [12-15]. The clinical utility of these 3DMFMs has been questioned in the past as the first publications lacked detailed information for reproducibility or omitted to report evidence of reliability studies [7]. Currently, 3DMFMs are increasingly utilized, as shown by the increasing number of publications. This systematic review presents a qualitative appraisal of the evidence embodying the scientific credibility and clinical utility of 3DMFMs. The scientific credibility of published 3DMFMs is of primary relevance for clinicians and gait laboratories involved in the assessment of foot and lower limb dysfunctions.

\section{Methods}

\subsection{In- and exclusion criteria}

Studies published in English as full papers on 3DMFMs in healthy and pathological foot conditions were considered. The definitions of the other inclusion and exclusion criteria are described in Table 1.

\subsection{Search strategy}

The review of the literature was performed by selecting articles from Pubmed, CINAHL, Cochrane Library and Web of Science (until December 2009). Keywords were selected from MeSH terminology and consisted of the words 'foot', 'motion' and 'model'. The search was narrowed by combining the latter terms with following search strings: 'kinematics', 'locomotion', 'repeatability', 'multisegmental foot model' and 'foot and ankle modelling'. Electronic searches were performed by one reviewer (K.D.), references and abstracts of studies were stored alphabetically into a separate worksheet. Additional relevant papers were identified by examining the reference lists of papers identified from electronic searching.

\subsection{Review process}

Duplicate references because of searches in different electronic databases were removed. Title and abstracts of the retrieved papers were subsequently evaluated for inclusion by two independent reviewers (G.M. and F.S.). A full text evaluation was performed if the title and abstract failed to provide adequate information. Any disagreement between the two reviewers was resolved by a consensus meeting. Finally, a second manual screening of the reference lists of all included studies was undertaken by one of the authors (K.D.), to affirm the inclusion of all eligible studies.

\subsection{Methodological quality assessment}

A systematic evaluation of scientific evidence must be done using a robust evaluation form based on expert guidelines [16,17]. The evaluation of the scientific credibility of 3DMFMs was based on quality indicators related to reproducibility, reliability and external validity. These indicators were tabulated in a quality form

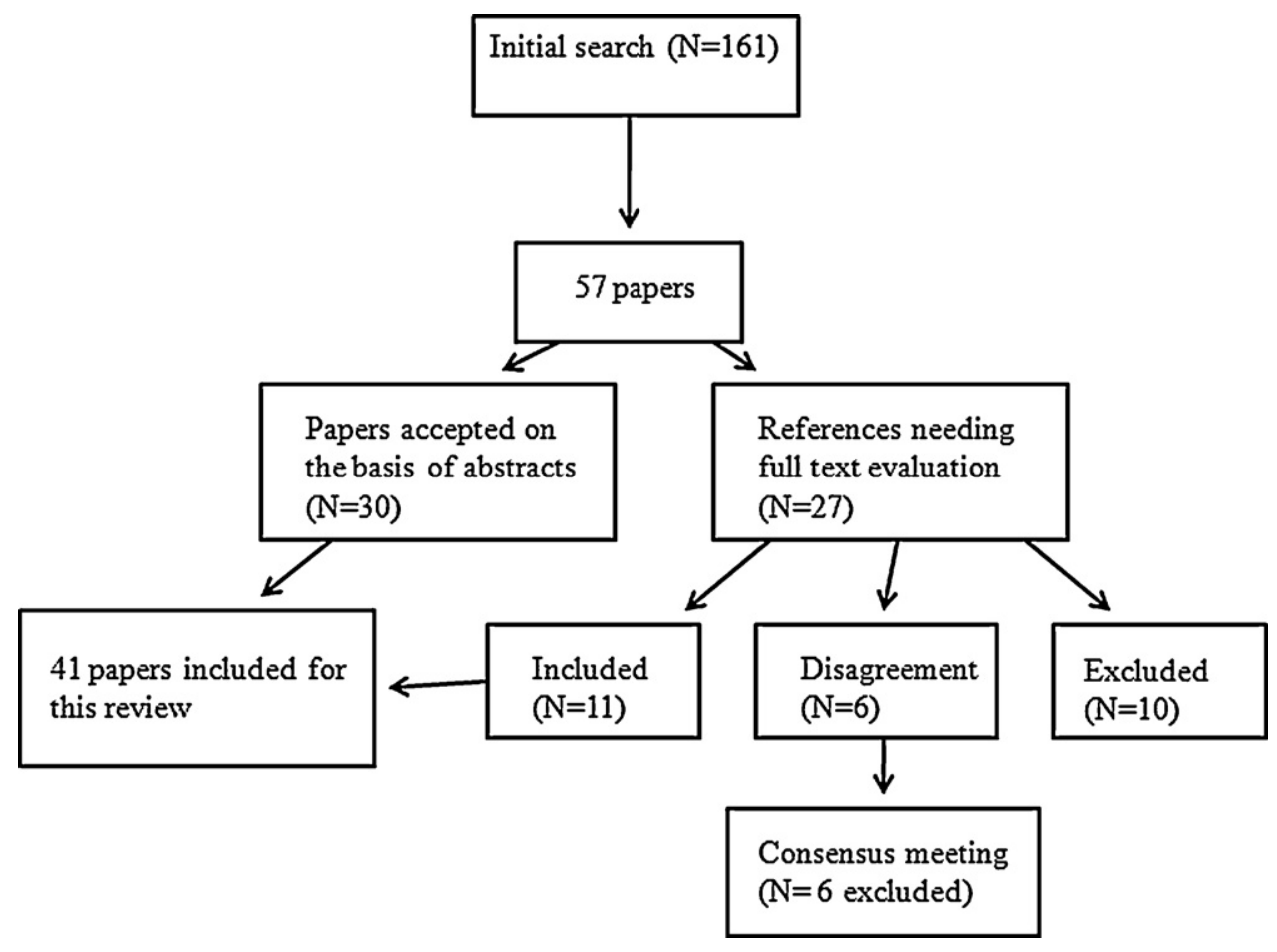

Fig. 1. Flowchart representing paper selection and reduction. 
(Scientific Credibility Form, SCF). The selection of these indicators was done by reviewing and selecting those qualitative parameters critical for an adequate 3D motion analysis research tool. The review by Capozzo et al. [18] was used to determine pertinent parameters in 3D motion representation, whereas the quality indicators related to repeatability and reproducibility were derived from three other papers [19-21]. The paper by McGinley et al. [21] served also as basis for the determination of quality indicators related to sample selection and study procedures. The quality indicators, selected by one clinician (K.D.), were not scored as there currently exists no relevant scoring system. All studies, including methodological and clinical papers, were qualitatively evaluated with the SCF.

\section{Results}

\subsection{Review selection and identification}

The initial search yielded 161 citations (Fig. 1). Thirty references were included based on the abstract. For 27 references the full text had to be reviewed of which 11 papers were included. Continuing disagreement existed on six papers [8,22-26] which were discussed during a consensus meeting. Three references $[22,23,25]$ were excluded as they did not model at least three segments. The narrative review of Rankine et al. [26] was excluded as well as the publication by Sawacha et al. [24], as no specific 3DMFM was used. The validity study performed by Nester et al. [8] was only used as background information for the introduction and discussion. Manual screening of the reference lists did not provide additional papers. As a result, 41 studies were identified for inclusion in the systematic review [27-67].

The references were grouped on the basis of the 3DMFM used. Fifteen different 3DMFMs could be distinguished (Table 2). The included studies were evaluated with the SCF in order to screen the quality indicators for each of the 3DMFMs. Results are summarized in Tables 3-5. The quality indicators related to sampling methods are shown in Table 3. Table 4 represents those indicators related to the study procedures. Marker placement associated with each model is presented in Additional file 1. Table 5 summarizes quality indicators assessing the reproducibility and experimental errors whereas Additional file 2 provides a best evidence synthesis regarding the reported repeatability of 3DMFMs.

\subsection{Sample selection, composition and description (Table 3)}

A majority of the papers mentioned the application of in- and exclusion criteria. Of those that did mention the use of inclusion and exclusion criteria, only one did not specify the type of criteria (e.g. presence of deformities, type of disease, and specific ranges of BMI).

Only one study reported the sampling method for recruitment of the subjects [58] leading to the assumption that mostly a convenience sample was used. The quality of the demographic information was poor in 10 studies and not reported in three studies. This lack of information limits the generalisation to specific populations. The number of participants in each study ranged from 1 to 153 in the 41 studies. Only two models were used for various pathologies and by various research labs, namely the Oxford Foot Model and the Milwaukee Foot Model. The clinical utility of 3DMFMs has been tested in several studies, with a majority of the papers focusing on tibialis posterior dysfunction. The participants in these studies varied widely, with studies presenting only one case [36] or studies including between 14 and 20 impaired persons $[35,57]$. Studies including the highest number of participants were characterized by cohorts of more than $30[33,63]$.

In studies with impaired participants other types of assessment tools or examinations were included (Table 3). Adequate

Table 2

Summary of published 3-D multisegment foot models.

\begin{tabular}{|c|c|c|c|}
\hline Specific name & Methodological study & Clinical studies & Modelled segments \\
\hline Model 1 (Milwaukee Foot Model) & $\begin{array}{l}\text { Kidder et al. [27] } \\
\text { Myers et al. [28] } \\
\text { Long et al. [29] }\end{array}$ & $\begin{array}{l}\text { Khazzam et al. [30] } \\
\text { Canseco et al. [31] } \\
\text { Khazzam et al. [32] } \\
\text { Ness et al. [33] } \\
\text { Canseco et al. [34] } \\
\text { Marks et al. [35] }\end{array}$ & Tibia/hindfoot/forefoot/hallux \\
\hline Model 2 & Rattanaprasert et al. [36] & & Tibia/rearfoot/forefoot/hallux \\
\hline Model 3 & Cornwall and McPoil $[37,38]$ & & Tibia/calcaneus/navicular/first metatarsal \\
\hline Model 4 & Leardini et al. [39] & Giacomozzi et al. [40] & Tibia/hindfoot/midfoot/first metatarsal/hallux \\
\hline $\begin{array}{l}\text { Model 5a: Oxford Foot } \\
\text { Model (2001) (Adults) }\end{array}$ & Carson et al. [41] & $\begin{array}{l}\text { Theologis et al. [42] } \\
\text { Woodburn et al. [43] } \\
\text { Turner et al. [44] } \\
\text { Turner et al. [45] }\end{array}$ & Tibia/Hindfoot/Forefoot/Hallux \\
\hline $\begin{array}{l}\text { Model 5b:Oxford Foot } \\
\text { Model (Children) (OFM) (2006) }\end{array}$ & $\begin{array}{l}\text { Stebbins et al. [46] } \\
\text { Curtis et al. [47] }\end{array}$ & Alonzo-Vazquez et al. [48] & Tibia/Hindfoot/Forefoot/Hallux \\
\hline Model 6 & Arampatzis et al. [49] & $\begin{array}{l}\text { Arampatzis et al. [50] } \\
\text { Morey-Klapsing et al. [51] } \\
\text { Morey-Klapsing et al. [52] } \\
\text { Morey-Klapsing et al. [53] }\end{array}$ & $\begin{array}{l}\text { Tibia/Talus/calcaneus/medial forefoot/lateral } \\
\text { forefoot/medial toes/lateral toes }\end{array}$ \\
\hline Model 7 & Mac Williams et al. [54] & & $\begin{array}{l}\text { Hallux/medial toes/lateral toes/medial } \\
\text { forefoot/lateral forefoot/calcaneus/cuboid/ } \\
\text { talus-navicular-cuneiform/tibia }\end{array}$ \\
\hline $\begin{array}{l}\text { Model 8: Heidelberg Foot } \\
\text { Measurement Method (HFMM) }\end{array}$ & Simon et al. [55] & Wolf et al. [56] & $\begin{array}{l}\text { Tibia/hindfoot/midfoot/forefoot/hallux/first } \\
\text { ray/fifth ray (+functional angles) }\end{array}$ \\
\hline Model 9 & Tome et al. [57] & $\begin{array}{l}\text { Rao et al. [58] } \\
\text { Nawoczenski et al. [59] }\end{array}$ & $\begin{array}{l}\text { Tibia/rearfoot/medial forefoot/lateral } \\
\text { forefoot/hallux ( }+1 \text { functional angle) }\end{array}$ \\
\hline Model 10 & Leardini et al. [60] & & $\begin{array}{l}\text { Tibia/Calcaneus/Mid-foot/metatarsus/hallux } \\
\text { (+ functional angles) }\end{array}$ \\
\hline Model 11 & Rao et al. [61] & & Tibia/calcaneus/lateral forefoot/first metatarsal \\
\hline Model 12 & Houck et al. [62] & Houck et al. [63] & Tibia/rearfoot/first metatarsal and hallux \\
\hline Model 13 & Jenkyn and Nicol $[64,65]$ & & Hindfoot/midfoot/medial and lateral forefoot \\
\hline Model 14 & Sawacha et al. [66] & & Tibia/Hindfoot/Midfoot/Forefoot \\
\hline Model 15 & Cobb et al. [67] & & $\begin{array}{l}\text { Rearfoot/calcaneonavicular/medial } \\
\text { forefoot/first metatarsal }\end{array}$ \\
\hline
\end{tabular}

a Each individual 3DMFM was given a random number and studies using the same 3DMFM were grouped together in order to facilitate the synthesis. 
Table 3

Summary of findings regarding quality indicators for sampling methods and description.

\begin{tabular}{|c|c|c|c|c|c|c|c|c|}
\hline Model & Study & $\begin{array}{l}\text { In- and exclusion } \\
\text { criteria }\end{array}$ & $\begin{array}{l}\text { Type of } \\
\text { criteria }\end{array}$ & $\begin{array}{l}\text { Sampling } \\
\text { method }\end{array}$ & $\begin{array}{l}\text { Quality of } \\
\text { demographic data }\end{array}$ & $\begin{array}{l}\text { Pathology specific } \\
\text { information }\end{array}$ & $\begin{array}{l}\text { Number of } \\
\text { participants (mean age) }\end{array}$ & $\begin{array}{l}\text { Assessor } \\
\text { characteristics }\end{array}$ \\
\hline \multirow[t]{9}{*}{1} & Kidder et al. [27] & A & A & NR & A & NA & $n=1$ HS $(25 y)$ & NR \\
\hline & Myers et al. [28] & NR & A & NR & NR & NA & $n=3$ HS $(6-11 y)$ & NR \\
\hline & Long et al. [29] & NR & NR & NR & NR & NA & $n=6$ HS $(21,2 y)$ & Experienced \\
\hline & Khazzam et al. [30] & A & A & NR & A & $\begin{array}{l}\text { CE, AOFAS score, } \\
\text { SF-36 HS }\end{array}$ & $\begin{array}{l}n=34 \text { AJA }(56 y) \\
n=25 \text { HS }(41 y)\end{array}$ & NR \\
\hline & Canseco et al. [31] & A & A & NR & A & CE, AOFAS & $\begin{array}{l}n=22(52 \mathrm{y}), \mathrm{HR} \\
n=25 \mathrm{HS}(41 \mathrm{y})\end{array}$ & NR \\
\hline & Khazzam et al. [32] & A & A & NR & A & CE, AOFAS & $\begin{array}{l}n=22 \mathrm{RA}(54 \mathrm{y}) \\
n=25 \mathrm{HS}(41 \mathrm{y})\end{array}$ & NR \\
\hline & Ness et al. [33] & A & A & NR & A & NR & $\begin{array}{l}n=34 \operatorname{TPD}(52,8 \mathrm{y}), \\
25 \mathrm{HS}(41,3 \mathrm{y})\end{array}$ & NR \\
\hline & Canseco et al. [34] & A & A & NR & A & CE, AOFAS & $\begin{array}{l}n=19 \operatorname{HR}(50,5 y) \\
25 \mathrm{HS}(41 \mathrm{y})\end{array}$ & NR \\
\hline & Marks et al. [35] & A & A & NR & A & NR & $n=20$ TPD $(52,4 y)$ & NR \\
\hline 2 & Rattanaprasert et al. [36] & A & A & NR & $\mathrm{P}$ & NR & $\begin{array}{l}n=1 \text { TPD }(35 y) \\
n=10 \text { HS }(30 y)\end{array}$ & NR \\
\hline 3 & Cornwall and McPoil $[37,38]$ & A & A & NR & A & NA & $n=153$ HS $(26,2 y)$ & NR \\
\hline \multirow[t]{2}{*}{4} & Leardini et al. [39] & A & A & NR & $P$ & NA & $n=9 \mathrm{HS}$ & NR \\
\hline & Giacomozzi et al. [40] & A & A & NR & $\mathrm{P}$ & MSS & $n=8$ TCC, $n=5$ HS (27y) & NR \\
\hline \multirow[t]{5}{*}{$5 a$} & Carson et al. [41] & NR & NR & NR & $\mathrm{P}$ & NA & $n=2$ HS $(26,5 y)$ & $\begin{array}{l}\text { Two } \\
\text { experienced }\end{array}$ \\
\hline & Theologis et al. [42] & NR & NR & NR & A & CE, PPQ & $\begin{array}{l}n=20 \operatorname{TEV}(9,8 \mathrm{y}), \\
15 \operatorname{HS}(10,7 \mathrm{y})\end{array}$ & NR \\
\hline & Woodburn et al. [43] & A & A & NR & A & SIP, SIS & $n=11 \mathrm{RA}, n=5 \mathrm{HS}$ & NR \\
\hline & Turner et al. [44] & A & A & NR & A & DAS, LFIS, SIS & $n=12 \mathrm{RA}, n=12 \mathrm{HS}$ & NR \\
\hline & Turner et al. [45] & A & A & NR & A & SIS & $\begin{array}{l}n=28 \text { RA }(58,7 y) \\
n=53 \text { HS }(55,2 y)\end{array}$ & NR \\
\hline \multirow[t]{3}{*}{$5 b$} & Stebbins et al. [46] & NR & NR & NR & $P$ & NA & $n=15$ HS $(9,5 y)$ & NR \\
\hline & Curtis et al. [47] & NR & NR & at random & NR & NA & $n=8$ HS $(12 y)$ & NR \\
\hline & Alonzo-Vazquez et al. [48] & NR & NR & NR & A & $\begin{array}{l}\text { Goniometric } \\
\text { evaluation }\end{array}$ & $\begin{array}{l}n=10 \text { FFV }(10,2 y) \\
n=11 \text { HS }(9,8 y)\end{array}$ & NR \\
\hline \multirow[t]{5}{*}{6} & Arampatzis et al. [49] & NR & NR & NR & $P$ & NA & $n=6$ HS $(21,2 y)$ & NR \\
\hline & Arampatzis et al. [50] & NR & NR & NR & $\mathrm{P}$ & NA & $n=6$ HS $(21,2 y)$ & NR \\
\hline & Morey-Klapsing et al. [51] & NR & NR & NR & A & NA & $n=22 \mathrm{HS}(26 \mathrm{y})$ & NR \\
\hline & Morey-Klapsing et al. [52] & NR & NR & NR & A & NA & $n=23 \mathrm{HS}(26 \mathrm{y})$ & NR \\
\hline & Morey-Klapsing et al. [53] & NR & NR & NR & $P$ & NA & $n=24 \mathrm{HS}$ & NR \\
\hline 7 & MacWilliams et al. [54] & A & A & NR & A & NA & $n=18 \mathrm{HS}(12,4 \mathrm{y})$ & NR \\
\hline \multirow[t]{2}{*}{8} & Simon et al. [55] & A & A & NR & $P$ & NA & $n=10 \mathrm{HS}(30 \mathrm{y})$ & NR \\
\hline & Wolf et al. [56] & A & A & NR & A & NA & $n=18$ HS $(8,2 y)$ & NR \\
\hline \multirow[t]{3}{*}{9} & Tome et al. [57] & A & A & NR & A & NR & $\begin{array}{l}n=14 \operatorname{TPD}(56,6 \mathrm{y}) \\
n=10 \mathrm{HS}(51,2 \mathrm{y})\end{array}$ & $\begin{array}{l}\text { Single } \\
\text { examiner }\end{array}$ \\
\hline & Rao et al. [58] & A & A & CS & A & FFI & $\begin{array}{l}n=30 \text { MFA }(62 \mathrm{y}) \\
n=20 \text { HS }(28 y)\end{array}$ & NR \\
\hline & Nawoczenski et al. [59] & A & A & NR & A & NR & $n=20$ HR $(49 y)$ & NR \\
\hline 10 & Leardini et al. [60] & A & A & NR & A & NR & $n=10 \mathrm{HS}(25,8 \mathrm{y})$ & Experienced \\
\hline 11 & Rao et al. [61] & A & A & NR & A & $\begin{array}{l}\text { Neurological } \\
\text { examination }\end{array}$ & $\begin{array}{l}n=15 \text { DM }(58 y) \\
n=15 \mathrm{HS}(56 \mathrm{y})\end{array}$ & NR \\
\hline \multirow[t]{2}{*}{12} & Houck et al. [62] & A & A & NR & A & NA & $n=21$ HS $(22 y)$ & NR \\
\hline & Houck et al. [63] & A & A & NR & A & NR & $\begin{array}{l}n=30 \operatorname{TPD}(59,8 \mathrm{y}) \\
n=15(56,5 \mathrm{y})\end{array}$ & NR \\
\hline \multirow[t]{2}{*}{13} & Jenkyn and Nicol [64] & A & A & NR & A & NA & $n=12 \mathrm{HS}$ & NR \\
\hline & Jenkyn and Anas [65] & A & A & NR & A & NA & $n=13 \mathrm{HS}$ & NR \\
\hline 14 & Sawacha et al. [66] & A & A & NR & A & $\begin{array}{l}\text { Neurological } \\
\text { examination }\end{array}$ & $\begin{array}{l}n=10 \text { DMN }(61,8 y), \\
n=10 \text { HS }(64 y)\end{array}$ & NR \\
\hline 15 & Cobb et al. [67] & A & A & NR & A & NA & $\begin{array}{l}n=11 \text { HS LMF }(24,5 y), \\
n=11 \text { HS }\end{array}$ & NR \\
\hline
\end{tabular}

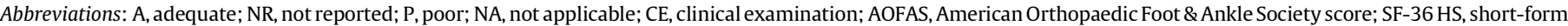

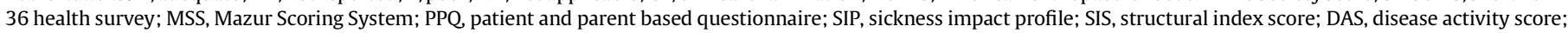

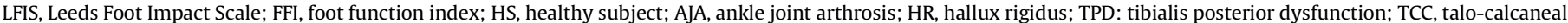

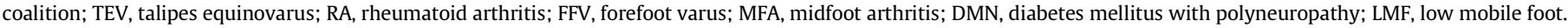

information about the assessors (e.g. background, experience, and age) was not available for the majority of the studies. Only two studies reported the number of assessors $[41,66]$. Three studies stated that the assessors were 'experienced' $[29,41,60]$, but without additional information about the background and level of experience.

\subsection{Study procedures (Table 4)}

All papers implied a specific measurement protocol with some using additional devices or methods. Some devices served to determine the neutral alignment of the foot and lower limb $[36,55,66]$. Others were used to test the accuracy of the motion analysis system (MAS) [54] or to track additional markers in the laboratory reference frame [39]. Patients were exposed to X-rays throughout all investigations using the Milwaukee Foot Model. The latter aimed at increasing the accuracy of the model, as the orientation of the markers to the bony anatomy was quantified by standard X-ray measurements.

For the majority of the models an optoelectronic motion capture system was used for tracking of passive reflecting markers. Four papers reported the use of an active system [57,61-63] and in 
Table 4

\begin{tabular}{|c|c|c|c|c|c|c|c|c|c|c|}
\hline Model & Study & $\begin{array}{l}\text { Use of additional } \\
\text { device }\end{array}$ & No. of markers & Sensors & $\begin{array}{l}\text { No. of cameras } \\
\text { (measurement frequency) }\end{array}$ & $\begin{array}{l}\text { Complete gait } \\
\text { cycle/stance phase }\end{array}$ & $\begin{array}{l}\text { Control of } \\
\text { events }\end{array}$ & $\begin{array}{l}\text { Number of } \\
\text { cycles }\end{array}$ & Speed & $\begin{array}{l}\text { Spatio-temporal } \\
\text { parameters }\end{array}$ \\
\hline \multirow[t]{4}{*}{1} & Kidder et al. [27] & Radiography $^{a}$ & $9+1$ triad & Pas & Five $(60 \mathrm{~Hz})$ & CGC & NR & $5-10$ & SSP & NR \\
\hline & Myers et al. [28] & & $9+1$ triad & Pas & $15(120 \mathrm{~Hz})$ & CGC & $\begin{array}{l}\text { Software } \\
\text { workspace }\end{array}$ & 9 & SSP & NR \\
\hline & Long et al. [29] & Radiography $^{\mathrm{a}}$ & Kidder et al. [27] & Pas & NR & CGC & $\begin{array}{l}\text { Software } \\
\text { workspace }\end{array}$ & NR & SSP & A \\
\hline & $\begin{array}{l}\text { Khazzam et al., Canseco et al., } \\
\text { Khazzam et al., Ness et al., } \\
\text { Canseco et al., Marks et al. [30-35] }\end{array}$ & Radiography $^{\mathrm{a}}$ & $9+1$ triad & Pas & $15(120 \mathrm{~Hz})$ & CGC & NR & 3 & SSP & A \\
\hline 2 & Rattanaprasert et al. [36] & Calliper $^{\mathrm{a}}$ & 10 markers & Pas & $4(60 \mathrm{~Hz})$ & SP & FP & 5 & SSP & NR \\
\hline 3 & Cornwall and McPoil $[37,38]$ & 1 & 4 & EMS & $\begin{array}{l}\text { Electromagnetic } \\
\text { transmitter }(60 \mathrm{~Hz})\end{array}$ & SP & FS & 5 & SSP & $\mathrm{L}$ \\
\hline \multirow[t]{2}{*}{4} & Leardini et al. [39] & CAST method ${ }^{a}$ & 5 rigid plates & Pas & $2(100 \mathrm{~Hz})$ & SP & FP & 3 & SSP & NR \\
\hline & Giacomozzi et al. [40] & CAST method ${ }^{a}$ & 4 rigid plates & Pas & NR $(100 \mathrm{~Hz})$ & SP & FP & NR & NR & NR \\
\hline \multirow[t]{4}{*}{$5 a$} & Carson et al. [41] & a jig & $15+2$ stick markers & Pas & $6(\mathrm{NR})$ & SP & FP & 10 & SSP & NR \\
\hline & Theologis et al. [42] & $\begin{array}{l}\text { Refers to Carson } \\
\text { et al. [41] }\end{array}$ & CGC & $\mathrm{FP}$ & 3 & SSP & A & & & \\
\hline & Woodburn et al. [43] & 1 & $16+2$ stick markers & Pas & $6(60 \mathrm{~Hz})$ & SP & FP & 5 & SSP & NR \\
\hline & Turner et al. $[44,45]$ & 1 & 22 & Pas & $6(60 \mathrm{~Hz})$ & SP & FP & 5 & NR & A \\
\hline \multirow[t]{3}{*}{$5 b$} & Stebbins et al. [46] & 1 & 17 & Pas & $12(100 \mathrm{~Hz})$ & CGC & NR & 3 & SSP & NR \\
\hline & Curtis et al. [47] & l & 18 & Pas & NR (NR) & CGC & NR & 3 & NR & A \\
\hline & Alonzo-Vazquez et al. [48] & 1 & $\begin{array}{l}\text { Refers to Stebbins } \\
\text { et al. [46] }\end{array}$ & Pas & $6(100 \mathrm{~Hz})$ & CGC & NR & 5 & NR & A \\
\hline \multirow[t]{5}{*}{6} & Arampatzis et al. [49] & 1 & 18 & Pas & $3(250 \mathrm{~Hz})$ & Barefoot landing & $\begin{array}{l}\text { Paromed } \\
\text { insoles }\end{array}$ & NR & Landing & NR \\
\hline & Arampatzis et al. [50] & 1 & 18 & Pas & $3(250 \mathrm{Jz})$ & $\begin{array}{l}\text { Barefoot landing } \\
(1.0 \mathrm{~m}, 1.5 \mathrm{~m}, 2 \mathrm{~m})\end{array}$ & NR & NR & Landing & NR \\
\hline & Morey-Klapsing et al. [51] & 1 & 17 & Pas & $4(120-250 \mathrm{~Hz})$ & Lateral tilts & Platform & NR & Self-defined & NR \\
\hline & Morey-Klapsing et al. [52] & 1 & 17 & Pas & $4(120-250 \mathrm{~Hz})$ & Medial-lateral tilts & Platform & 3 & self-defined & NR \\
\hline & Morey-Klapsing et al. [53] & i & 17 & Pas & $4(120-250 \mathrm{~Hz})$ & One leg drop landings & FP & 3 & Landing & A \\
\hline 7 & MacWilliams et al. [54] & wedges $^{\mathrm{b}}$ & 19 & Pas & $6(60 \mathrm{~Hz})$ & SP & FP & 5 & SSP & NR \\
\hline \multirow[t]{2}{*}{8} & Simon et al. [55] & $\begin{array}{l}\text { Heel alignment } \\
\text { device }^{\mathrm{a}}\end{array}$ & 17 & Pas & $6(50 \mathrm{~Hz})$ & CGC & $\mathrm{NR}$ & 8 & SSP & NR \\
\hline & Wolf et al. [56] & 1 & $\begin{array}{l}\text { Subset of markers } \\
\text { (Simon et al. [55]) }\end{array}$ & Pas & $6(120 \mathrm{~Hz})$ & CGC & & & SSP & A \\
\hline \multirow[t]{3}{*}{9} & Tome et al. [57] & 1 & $\begin{array}{l}5 \text { rigid plates }+1 \\
\text { single IRED }\end{array}$ & ACT & $6(60 \mathrm{~Hz})$ & SP & FP & 5 & SSP & NR \\
\hline & Rao et al. [58] & 1 & 5 & EMS & $\mathrm{NR}$ & $\begin{array}{l}\text { walking and step } \\
\text { descent task }\end{array}$ & NR & NR & NR & NR \\
\hline & Nawoczenski et al. [59] & 1 & 3 & EMS & $100 \mathrm{~Hz}$ & SP & NR & 3 & SSP & NR \\
\hline 10 & Leardini et al. [60] & i & 14 & Pas & $8(100 \mathrm{~Hz})$ & SP & FP & 3 & SSP & NR \\
\hline 11 & Rao et al. [61] & 1 & $9+1$ rigid plate & ACT & $3(120 \mathrm{~Hz})$ & SP & FP & 5 & $0,89 \mathrm{~m} / \mathrm{s}$ & $\mathrm{L}$ \\
\hline \multirow[t]{2}{*}{12} & Houck et al. [62] & I & 12 & ACT & $\begin{array}{l}\text { Active optical } \\
\text { system }(60 \mathrm{~Hz})\end{array}$ & SP & FP & 5 & SSP & NR \\
\hline & Houck et al. [63] & 1 & 12 & ACT & $6(60 \mathrm{~Hz})$ & Heel rise test & FP & 6 & Heel rise test & 1 \\
\hline 13 & $\begin{array}{l}\text { Jenkyn and Nicol [64], } \\
\text { Jenkyn and Anas [65] }\end{array}$ & i & 7 rigid clusters & ACT & $6(50 \mathrm{~Hz})$ & CGC & NR & 5 & SSP & NR \\
\hline 14 & Sawacha et al. [66] & Footguide $^{\mathrm{a}}$ & 13 & Pas & $6(60 \mathrm{~Hz})$ & CGC & FP & 3 & SSP & $\mathrm{L}$ \\
\hline 15 & Cobb et al. [67] & 1 & $20+1$ cluster & Pas & $8(120 \mathrm{~Hz})$ & SP & FP & $3-5$ & NR & NR \\
\hline
\end{tabular}

Abbreviations: Pas, passive; EMS, electromagnetic sensor; ACT, active; CGC, complete gait cycle; SP, stance phase; NR, not reported; FP, force plate; FS, force sensor; SSP, self-selected speed; A, adequate; L, limited.

a Additional devices used during the testing procedure (e.g. to determine foot position, help with marker placement and anatomical calibration).

Equipment used to perform error assessment. 


\begin{tabular}{|c|c|c|c|c|c|c|c|}
\hline \multirow[t]{2}{*}{ Model } & \multirow[t]{2}{*}{ Study } & \multicolumn{4}{|c|}{ Reproducibility of the model } & \multicolumn{2}{|l|}{ Experimental errors } \\
\hline & & $\begin{array}{l}\text { Modelled } \\
\text { segments }\end{array}$ & $\begin{array}{l}\text { Location and } \\
\text { orientation of } \\
\text { anatomical } \\
\text { reference frames }\end{array}$ & $\begin{array}{l}\text { Method of } \\
\text { 3D orientation } \\
\text { description }\end{array}$ & Reference position & MAS accuracy & $\begin{array}{l}\text { Repeatability of } \\
\text { gait measurements }\end{array}$ \\
\hline \multirow[t]{4}{*}{$1^{\mathrm{a}}$} & Kidder et al. [27] & 4 & $\mathrm{~L}$ & Euler & $\begin{array}{l}\text { Roentographic correction } \\
\text { procedure }\end{array}$ & $\begin{array}{l}\text { Residual value, Static and } \\
\text { dynamic tests }\end{array}$ & NR \\
\hline & Myers et al. [28] & & & & & Static and dynamic tests & NR \\
\hline & Long et al. [29] & & & & & NR & $\begin{array}{l}\text { Intra- Inter session variability for each subject within-and } \\
\text { between sites)/Intra- inter subject across all sites (CMC) }\end{array}$ \\
\hline & Khazzam et al. [30] & & & & & NR & intra-subject variation (COV) \\
\hline 2 & Rattanaprasert et al. [36] & 4 & NR & $\mathrm{JCS}^{\mathrm{a}}$ & $\begin{array}{l}\text { Calliper/positional alignment/ } \\
\text { calcaneal inclination to } \\
\text { compute zero position }\end{array}$ & Dynamic test & $\begin{array}{l}1 \text { clinician } 4 \text { normal subjects } 2 \text { separate days/ } \\
\text { day-to-day and trial-to-trial reliability (CMC) }\end{array}$ \\
\hline 3 & Cornwall and McPoil $[37,38]$ & 4 & A & $\mathrm{JCS}^{\mathrm{a}}$ & Relaxed position & NR & Within-subject reliability (SD, SEM, CMC) \\
\hline \multirow[t]{2}{*}{4} & Leardini et al. [39] & 5 & A & $\mathrm{JCS}^{\mathrm{a}}$ & $\begin{array}{l}\text { Standing upright posture } \\
(+ \text { considered as offset value) }\end{array}$ & Static and dynamic test & Intra- and inter-subject repeatability (CMC) \\
\hline & Giacomozzi et al. [40] & $\begin{array}{l}\text { Refers to } \\
\text { Leardini et }\end{array}$ & al. [39] & & & Spot check procedure & NR \\
\hline \multirow[t]{3}{*}{$5 a^{b}$} & Carson et al. [41] & 4 & A & $\mathrm{JCS}^{\mathrm{a}}$ & Static trial using a jig & Calibration residual & $\begin{array}{l}\text { Systematic/between-trial/between-day/between tester } \\
\text { variability/2 testers, } 2 \text { healthy subjects, } 4 \text { days (SD) }\end{array}$ \\
\hline & Woodburn et al. [42] & $\begin{array}{l}\text { Refers to } p \\
\text { of Carson }\end{array}$ & $\begin{array}{l}\text { otocol } \\
\text { al. }[41]\end{array}$ & & & NR & $\begin{array}{l}\text { Within-day variability for control group and RA } \\
\text { group, between-day variability only for control (CMC) }\end{array}$ \\
\hline & Turner et al. $[44,45]$ & $\begin{array}{l}\text { Refers to } p \\
\text { Carson et } a\end{array}$ & $\begin{array}{l}\text { otocol of } \\
\text { [41] }\end{array}$ & & & NR & Inter-subject variability of waveforms (mean $\pm \mathrm{SD}$ ) \\
\hline \multirow[t]{3}{*}{$5 b$} & Stebbins et al. [46] & 4 & A & $\mathrm{JCS}^{\mathrm{a}}$ & Static trial & NR & $\begin{array}{l}\text { Within-and between-day repeatability/15 children, } \\
3 \text { test sessions (SD) }\end{array}$ \\
\hline & Curtis et al. [47] & $\begin{array}{l}\text { Refers to } p \\
\text { Stebbins et }\end{array}$ & $\begin{array}{l}\text { otocol of } \\
\text { al. [46] }\end{array}$ & & & NR & Intra-rater repeatability (statistic: SD, ICC and TEM) \\
\hline & Alonzo-Vazquez et al. [48] & $\begin{array}{l}\text { Refers to } p \\
\text { Stebbins et }\end{array}$ & $\begin{array}{l}\text { otocol of } \\
\text { al. [46] }\end{array}$ & & & NR & Inter-subject variability of waveforms (mean $\pm \mathrm{SD}$ ) \\
\hline $6^{c}$ & Arampatzis et al. [49] & 7 & $\mathrm{P}$ & $\mathrm{JCS}^{\mathrm{a}}$ & Mechanic frame/seated position & NR & Inter-subject variability of waveforms (mean \pm SD) \\
\hline 7 & Mac Williams et al. [54] & 9 & $\mathrm{~L}$ & Euler & Foot in plantigrade position & Static test & Intra- and inter-subject variability (SD, CMC) \\
\hline \multirow[t]{2}{*}{8} & Simon et al. [55] & 7 & A & Projection angles & $\begin{array}{l}\text { Static trial/using heel } \\
\text { alignment device }\end{array}$ & NR & $\begin{array}{l}\text { Inter-tester, inter-day and inter-trial reliability/one } \\
\text { subject, five testers (SD, CMC) }\end{array}$ \\
\hline & Wolf et al. [56] & $\begin{array}{l}\text { Refers to S } \\
\text { a sub set o } \\
\text { was used }\end{array}$ & $\begin{array}{l}\text { mon et al. [55], } \\
\text { markers }\end{array}$ & & & NR & Inter-subject variation (SEM) \\
\hline $9^{\mathrm{d}}$ & Tome et al. [57] & 5 & $\mathrm{~L}$ & Euler & Subtalar neutral position & NR & Inter-subject variability of waveforms (mean \pm SD) \\
\hline 10 & Leardini et al. [60] & 5 & A & $\mathrm{JCS}^{\mathrm{a}}$ & Static trial & NR & Inter-subject variability of waveforms (mean \pm SD) \\
\hline 11 & Rao et al. [61] & 4 & A & Euler & $\begin{array}{l}\text { Stance phase mean used } \\
\text { as off-set }\end{array}$ & NR & Inter-subject variability of waveforms (mean \pm SD) \\
\hline $12^{\mathrm{e}}$ & Houck et al. [62] & 4 & A & Cardan angles & Compared RCSP and NCSP & NR & $\begin{array}{l}\text { Reliability of neutral positioning (ICC SEM and } \\
\text { absolute error) }\end{array}$ \\
\hline 13 & Jenkyn and Nicol $[64,65]$ & 4 & A & $\mathrm{JCS}^{\mathrm{a}}$ & Static standing position & Camera calibration residual & Within- and between subject repeatability (CMC) \\
\hline 14 & Sawacha et al. [66] & 4 & A & Cardan angles & Footguide & NR & $\begin{array}{l}\text { Inter-trial/Inter-day and Inter-session variability for } \\
\text { healthy and pathological group (SD) }\end{array}$ \\
\hline 15 & Cobb et al. [67] & 4 & A & $\mathrm{JCS}^{\mathrm{a}}$ & Seated position & Camera calibration residual & Within-session CMC \\
\hline
\end{tabular}

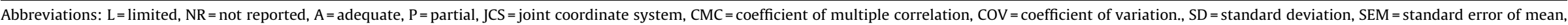
ICC $=$ intraclass correlation coefficient.

${ }^{a}$ Model 1: Canseco et al. [31], Khazzam et al. [32], Ness et al. [33], Canseco et al. [35], Marks et al. [35] provided no additional information.

b Model 5a: Theologis et al. [42] provided no additional information.

c Model 6: Arampatzis et al. [49], Morey-Klapsing et al. [51], Morey-Klapsing et al. [53] provided no additional information.

d Model 9: Rao et al. [58], Nawoczenski et al. [59] provided no additional information.

e Model 12: Houck et al. [63] provided no additional information. 
three studies an electromagnetic system was used [37,38,61]. The number of markers and cameras used was adequately provided in all studies. The majority of studies reported a specific gait protocol with approximately half of the investigators analysing stance phase of gait and allowing a self-selected speed during walking. One study selected a fixed walking speed [61] and six studies focused on other functional tasks such as landing and descent walking [49-53,58]. In most of the cases force plates have been used to determine gait events. Nine papers did not clearly indicate how gait events were determined.

Three trials was found to be the minimum number of recordings; however, most of the investigators recorded up to five or 10 trials. With respect to the data analysis, it was often unclear how many and which trials were used. Some studies omitted to report the number of trials used $[29,40]$, others only reported the number of trials recorded [27,28,36-38,43,57,60], several clearly indicated how many trials were used for statistical analysis $[30-35,39,46]$ and others specified that a mean or median was calculated $[41,44,45,48,55]$.

\subsection{Reproducibility and experimental errors}

\subsubsection{Reproducibility (Table 5)}

3.4.1.1. Modelled segments/Marker placement (Table 5 and Additional file 1). Most 3DMFMs considered three or four segments including the hindfoot (rearfoot), midfoot, forefoot and hallux. An adequate description of the segments and associated marker placement was reported for all models. MacWilliams et al. [54] differentiated the highest number of segments (nine segments) in their 3DMFM.

A majority of the 3DMFMs used at least three non-collinear markers to represent each segment (Additional file 1). Often single markers were used exclusively. Some authors used rigid plates alone, or a combination of both approaches, as it was believed to facilitate the motion tracking. It should be noted that some markers were only used during static trials and that Leardini et al. [39] have complemented their marker set with the CAST method [68].

The tibia was considered as the proximal segment for the motion analysis at the ankle being a rigid segment including the tibia and fibula and marked by three to seven markers $[37,38,55]$.

In a majority of the publications the calcaneus and talus were modelled as a single segment with both bones represented by markers placed on the calcaneus. Different marker setups have been proposed with five markers being the maximum number used [46]. This segment is often referred as rearfoot or hindfoot. Five investigators considered the midfoot as a separate segment and the marker set used for modelling varied considerably [39,60,64,66] (Additional file 1).

The forefoot was often defined as one rigid segment, including the five metatarsals. Three studies divided the forefoot into a medial and lateral segment: Jenkyn and Nicol $[64,65]$ used the first and fifth metatarsals, Tome et al. [57] the first and second to fourth metatarsal and MacWilliams et al. [54] omitted to provide adequate specifications. Arampatzis et al. [49] also modelled the lateral and medial forefoot, but included bones from the midfoot.

The first metatarsal was considered as an independent segment by Leardini et al. [60] and Houck et al. [62], while the lateral part of the forefoot was not considered in these models. A comparable approach was observed in the publication of Cornwall and McPoil [37].

The hallux has been considered in a majority of the models having 1, 2 or 3 degrees of freedom. Due to its relatively small size, authors have chosen to use only one marker or a triad of markers. Kinematics of the lateral toes was modelled by two authors [50,54].
In order to facilitate the modelling, it was observed that some segments of the 3DMFM shared landmarks, such as the lateral and medial forefoot segments of Jenkyn and Nicol [64], as well as the forefoot and midfoot by Leardini et al. [60].

3.4.1.2. Location and orientation of anatomical reference frames (Table 5). The level of description of the location and orientation of the anatomical reference frames (ARFs) of the modelled segments varied considerably. Some papers omitted to report the ARF for the different segments [36,54,57], while others only gave a description for a minority of the segments $[27,49]$. However, for the majority of the models an adequate description of the anatomical reference frame (including orientation of the $x, y, z$ axes) was provided and these therefore have the potential to be most reproducible $[39,41,46,55,60-62,64,66]$. The use of radiographs (Milwaukee Foot Model) [27-35] or digitized points [39] has been used to improve the identification of the ARF. A deviation from the standard approach of using at least 3 non-collinear markers to determine the ARF was observed in the Heidelberg Foot Measurement Method (HFMM) [55]. The HFMM has been called 'a method' instead of a 'model', because it does not use a formal definition of segments. A segment is represented as a vector, determined by at least 2 markers.

Non-uniformity in the definition of the ARFs of the different segments was observed throughout the 3DMFMs. This phenomenon was most evident in the midfoot segment [39,60,64], as well in the forefoot segment $[54,57,64]$ precluding the simple comparison of the kinematic measurements.

3.4.1.3. Method of 3-D orientation description (Table 5). A description of the method employed for joint angle representation was provided adequately in all publications. It can be observed from Table 5 that Cardan/Euler angles or the joint coordinate system (JCS) are used as method for 3-D orientation description in the majority of cases.

In the HFMM developed by Simon et al. [55], projection angles are used to represent the motion between two segments. Here, motion is described as relative motion between two simple vectors along a predetermined axis of rotation which does not necessarily coincide with the standard planes of reference.

Two distinct approaches were taken to the description of 3-D motion. In the first approach the motion between adjacent segments is calculated. This inter-segmental angle is then often linked to a specific joint and/or to the functional anatomy of the foot. In the second approach, motion between non-adjacent segments (e.g. forefoot and tibia, first metatarsal and rearfoot) is modelled. Such an approach was used exclusively by Cornwall and McPoil [37], who described the motion of the calcaneus, navicular and first metatarsal with respect to the tibia. Finally, several authors have also modelled some 'clinical' (sometimes referred as 'functional') angles believed to be of clinical interest in underpinning specific biomechanical foot concepts. As such, the medial longitudinal arch has been modelled by several authors $[46,55,57,60,64]$. Focusing on the validation of a multi-segmental foot model for children, Stebbins et al. [46] expressed the arch height with respect to the percentage of foot length. Other functional angles that have been modelled from a clinical viewpoint are the hallux valgus angle, first intermetatarsal angle, the planar angle of the first-second and fifth metatarsal, metatarsal 1-metatarsal 5 angle, lateral arch and medial arch inclination angle [55,60]. Stebbins et al. [46] included the inter-segmental angle between the forefoot and tibia in order to consider their findings in light of the conventional lower limb models and clinically interpret the motion between the hindfoot and tibia and forefoot and hindfoot. 
3.4.1.4. Reference position (Table 5). The description of the reference position used to interpret the kinematical waveforms was generally well detailed; however, a wide variety of methods and approaches were observed (Table 5). Several authors have defined the 'reference' position of the foot and leg as the simple comfortable standing position of the participant $[36,36,39,60]$. In one study [36], the reference position of all segments (with exception for the hindfoot inversion/eversion) was referenced using a simple static standing position. For the inclination of the hindfoot, a calliper was used in order to calculate the angle to which the hindfoot (bisection line) deviates from a vertical line which was subsequently used to compute the zero position. From the reference position, an offset value was calculated and subsequently subtracted from the motion data. In the study of Houck et al. [62] the major focus was to evaluate the repeatability of their protocol developed to determine the zero alignment (neutral position of the subtalar joint). The positioning of the subtalar joint in their neutral alignment could be performed in a good repeatable way in healthy subjects. Others have used an imposed position (vertical tibia) or complemented their protocol with specific devices such as a jig or a heel alignment device $[41,46,55]$. For these latter models, no offset values were subtracted from the recorded kinematic waveforms.

\subsubsection{Experimental errors/repeatability (Table 5)}

3.4.2.1. Motion analysis system (MAS) accuracy. The evaluation of procedural errors coming from the measurement equipment varied markedly across the different papers. Four studies have reported the procedural error related to the reconstruction of markers $[27,41,64,67]$.

Some authors specifically reported the accuracy of their motion analysis system. Kidder et al. [27] reported an excellent accuracy (fraction of the calibrated field of view $=0.083 \%$ ) of their Vicon motion analysis system under static and dynamic circumstances, using respectively a rigid steel rod with two markers and a pendulum. Rattanaprasert et al. [36] used a two-segment model which was manipulated with wedges in order to simulate movements in the three planes. Satisfactory results were obtained as the smallest range of motion was almost 7 times larger than the mean error. Leardini et al. [39] referred to a previously conducted stereophotogrammetric system validation study which used a mechanical simulator for test precision and accuracy $(0.3 \mathrm{~mm}$ root mean square for small calibrated field of view). Giacomozzi et al. [40] tested the precision and accuracy of their MAS using the socalled spot check procedure and found it to be less than $2 \mathrm{~mm}$. MacWilliams et al. [54] evaluated model accuracy by applying wedges under the feet of the participants. An averaged mean error of $1.4^{\circ}$ was found for the three ankle angle settings, whereas for two separate angles of toe extension, the averaged mean error was found to be $1.6^{\circ}, 3.3^{\circ}$ and $1.8^{\circ}$ for respectively the hallux, medial toes and the lateral toes. Myers et al. [28] used a Biodex System for dynamic angular testing and a dummy segment for performing the linear testing. Both tests showed adequate resolution and the MAS was found to be adequate for motion tracking.

3.4.2.2. Repeatability of kinematic measurements (Table 5). Several investigators provided some form of reliability index in their initial investigation (Additional file 2). Most often, coefficient of multiple correlation (CMC), or coefficient of multiple determination (CMD) were used in order to examine the consistency across the kinematic waveforms. Absolute measures of variation to describe the inter-day $[41,46,55,66]$, inter-trial $[55,66]$ and inter-session variabilities $[41,55,66]$ of the inter-segmental motion were: the range of motion, the standard deviation (SD), standard error of the measurement and the mean.
A 'best evidence synthesis' [69] regarding the repeatability of the reported 3DMFMs is provided, as a meta-analysis of the results was precluded due to the diversity in the studies (Additional file 2). To provide a qualitative indication and to simplify the synthesis, three categories were created. Category A summarizes the models where only the inter-subject variability of the kinematic waveforms has been reported (mean and \pm SD bands) (models 6, 9, 10 and 11). Category B summarizes the models which report repeatability indices of one test session only. It should be emphasized that both categories provide only an indication of the repeatability as they include only normal subjects. Regarding the models published by Cornwall and McPoil [37,38] and Cobb et al. [67] respectively model 3 and 15, CMC and SD values are available for the inter-subject variability. Cornwall and McPoil [37,38] found CMC values between 0.775 and 0.965 for their trial-to-trial analysis and SD values less than $0.36^{\circ}$. The segment with the biggest variation was found to be the first metatarsal (inversion/ eversion). The functional articulations of the model published by Cobb et al. [67] showed all CMC values above 0.83 .

The models 4,7 and 13 presented evidence not only from interbut also from intra-subject (normal subjects) repeatability indices. Leardini et al. [39] reported intra-subject CMC values between 0.64 and 0.95, MacWilliams et al. [54] reported values 0.57 and 0.93 , whereas for Jenkyn and Nicol [64] reported values between 0.58 and 0.92. Typically, the inter-subject CMC values were found to show more variability, with CMC values between 0.03 and 0.65 for model $4,0.11$ and 0.82 for model 7 and between 0.31 and 0.71 for model 13. Evaluation of these inter-subject CMC values shows that sagittal plane kinematics is facing the highest repeatability. MacWilliams et al. [54] also reported SD values for sagittal plane kinematics. The averaged SD values ranged between $0.8^{\circ}$ and $4.0^{\circ}$ for the intra-subject analysis, whereas these values ranged between $4.8^{\circ}$ and $11.1^{\circ}$ when inter-subject analyses were considered.

Houck et al. [62] reported repeatability indices regarding the reference positioning. These authors not only reported the between-subject mean kinematic waveforms with their associated $\mathrm{SD}$, but also estimated the repeatability of positioning the subtalar joint in its neutral position as the basis for the determination of the reference position. Intraclass correlation coefficients were found to be above 0.95 for all segments and the SEM statistic predicted errors less than $1.4-2.4^{\circ}$ in $96 \%$ of the cases.

Finally, category C summarizes those 3DMFMs reporting indices from more than 2 components of variability. Here, repeatability indices were obtained from multiple test sessions, different test days and different assessors.

The model proposed by Rattanaprasert et al. [36] was found to have a good reliability from trial to trial and day-to-day. Here, data were collected from four normal subjects on two separate days; however, no specifications were given about the number or the experience of the assessors. For all segments under investigation, except the forefoot-rearfoot inversion/eversion, high correlations were found between trials and between days.

Sawacha et al. [66] quantified the inter-day ( 1 healthy subject/1 assessor/2 sessions/3 trials), inter-trial ( 1 healthy subject/ 1 day/ 1 assessor/3 trials) and inter-session ( 1 healthy subject/5 assessors/3 trials) variability for their model. In order to do so, not only the mean, range and SD were quantified, but also the absolute variability (Vabs), a measure of discordance described by Noonan et al. [70] was reported. The latter represents the mean range of variability of a kinematic variable and is calculated by averaging the range of values for a specific number of points along the curve. The inter-trial SD values of all kinematic parameters ranged between $0.59^{\circ}$ and $1.22^{\circ}$, whereas higher SD values were reported for inter-day comparison (between $0.95^{\circ}$ and $2.89^{\circ}$ ). Repeatability analyses carried out on patients with diabetes mellitus yielded comparable results. 
Repeatability indices available for the Heidelberg Foot Measurement Method [55] are related to inter-tester, inter-day and inter-trial calculations including 10 healthy participants, five different testers and up to five sessions. Inter-trial CMC values ranged between 0.834 and 0.993 with associated SD values between $0.45^{\circ}$ and $1.51^{\circ}$. Inter-day CMC values were lower and ranged between 0.383 and 0.984 . Finally, inter-rater CMC values resulted in even worse results, with CMC values between 0.124 and 0.970. However, inter-rater CMC calculations were found to be affected by a varying offset rather than a variation in the shape of the waveforms. Modified inter-rater CMC values were therefore calculated demonstrating an overall better repeatability (between 0.508 and 0.980). Based on these findings, the authors were confident that the HFMM could be used for the assessment of clinical disorders.

Several studies shed some light on the repeatability of the Oxford Foot Model (model 5a and b).

Carson et al. [41] tested the trial-to trial, day-to-day and between tester variabilities including two clinicians and two healthy participants. The between-trial standard deviation was found to be the highest for the hallux-forefoot sagittal plane motion and tibia-floor transverse plane rotation $\left(1.45^{\circ}\right)$, however, both parameters were characterized by a ROM of more than $40^{\circ}$. Results from the between-tester analysis and between-day analysis were found to be similar and, as expected, also demonstrated higher variability. However, this decreased level of repeatability was primarily caused by a shift in absolute value of the intersegment angle, rather than a different waveform of the curves.

Woodburn et al. [43] estimated the within and between day repeatability from five trials for their healthy volunteers taken on 2 separate days. For their pathological group (11 patients with RA), only the within day repeatability could be assessed as only one gait analysis session was arranged for them. In this study, the betweenand within-day repeatability for all segments in all planes exceeded the 0.8 CMC value, with the exception of rearfoot internal/external rotation (CMC 0.6777). The studies by Turner et al. $[44,45]$ have provided additional data supporting the use of the Oxford Foot Model in patients with rheumatoid arthritis. These authors reported a distinct kinematic pattern in all segments which was qualitatively represented with mean kinematic waveforms together with SD bands.

Stebbins et al. [46] adapted the Oxford Foot Model (model 5a) developed by Carson et al. [41] in order to use it with children. The adaptations were mainly focused on the displacement or the elimination of single markers and the impact of these changes was tested in fifteen children three separate occasions. The proposed modifications did not significantly alter the inter-segmental motion patterns. A general trend for some offsets in the waveforms between days was demonstrated. Sagittal plane kinematics was found to be the most repeatable and the highest variability was observed in the transverse plane which was comparable to the results obtained with the classical Oxford Foot Model (2001). Based on these results, the authors were confident that the original model could be used in children.

Curtis et al. [47] evaluated the absolute and relative repeatability of the Oxford Foot Model [46] at each stage of the foot roll over process within a cohort of eight children. Foot motion was recorded on two separate days and this within a time span of 1 month. It was unclear how many assessors participated to this study. Kinematic patterns found in this study were consistent with those initially presented by Stebbins et al. [46], however, considerable offsets in absolute joint angles were reported. Repeatability was found to be stable throughout the gait cycle, with no major alterations occurring at the three functional rockers of gait. The most repeatable patterns occurred again in the sagittal plane whereas the transverse plane demonstrated the poorest repeatability. The absolute reliability was for most of the parameters $<4^{\circ}$, and this for the three rockers. Absolute measures of hindfoot internal rotation were found to be very variable (maximum difference $8.6^{\circ}$ ) but consistent throughout the gait cycle.

Variability and the repeatability of the Milwaukee Foot Model were investigated in a multi-center study including six healthy adults and multiple test sessions [29]. Inter-subject CMC values for between and within-sessions measurements were found to be $>0.77$. A comparable finding was observed for the within-subject calculations. However, the between-subject comparison was found to demonstrate the highest variability and this across all segments and planes of motion. Measurement error associated with site and session was less than $3.5^{\circ}$ in over $80 \%$ of position measurements and less than $2.5^{\circ}$ in over $80 \%$ of range of motion measurements. The authors concluded that the results of their study support the continued use of the Milwaukee Foot Model in different gait laboratories.

\section{Discussion}

The present review aimed to inform the healthcare provider regarding the scientific value of published 3DMFMs. Quality indicators were predetermined, assessing the reproducibility, repeatability and external validity of the published 3DMFMs. Furthermore, the standardization level was investigated. The wide variety in biomechanical modelling, methods, study participants and study design precluded a simple synthesis and a more quantitative assessment of the different models by means of metaanalysis. A best evidence synthesis was therefore proposed.

A detailed description of a new tool such as 3DMFMs is essential for allowing an adequate interpretation of the outcome measures and for reproduction by others. In general, the reproducibility of the 3DMFMs could be classified as good. A majority of studies clearly described the anatomical location of the markers, the anatomical reference frames, reference position and the method of intersegmental angle calculation. This allows the implementation in other laboratories in order to further validate the models and investigate their clinical utility. In five models reproduction was precluded as no detailed description of the ARF was provided $[27,36,49,54,57]$

An adequate description of the study procedures is of primary importance not only for those who want to reproduce the study, but also for the clinicians who want to implement a 3DMFM in their routine clinical practice. Even though a majority of the studies described to a satisfactory level their measurement procedures, some important differences were observed. The number of cameras, the specific motor task under investigation and the use of additional devices varied between studies. These elements are of importance if an adequate implementation of a specific 3DMFM is pursued.

Another noteworthy difference observed in the current review is the fact that some studies used an electromagnetic system whereas others used a retro-reflective optical system. At present, it is unknown which system is most optimal to represent the anatomical reference frame of a specific segment, especially when this segment includes different bones or if this segment has a complex orientation. Moreover, it is unclear which system is optimal in view of developing a repeatable and reproducible 3DMFM.

Estimating both intrinsic and extrinsic sources of errors typically encountered in 3D gait analysis forms the basis for the clinical validation of an assessment tool [20].

The first step in this process relates to estimating the reliability and measurement accuracy of the MAS used. For gait analysis 
purposes, the system validation protocol often includes static and dynamic tests. Only a minority of the investigators have recognized this vital issue and have, as such, reported a system accuracy study [27,28,36,39-41,54,64,67].

The second step in the validation process of an assessment tool consists of defining normative values in unimpaired and impaired persons. In this context, the application of the 3DMFM in a healthy population not only serves as feasibility test for typical feet, but also serves to gain reference data and, obviously, gains novel insight in foot (patho) mechanics. Findings from the literature demonstrate that the evaluation of healthy subjects has been successfully obtained for all 3DMFMs. In fact, all 3DMFMs reported typical kinematic patterns for the inter-segmental angles providing a discriminative basis for foot pathologies. Some studies have also found a 'typical' kinematical pattern for a number of pathologies. However, generalisation of results is only possible when an unbiased sample is used. The majority of the manuscripts did not stipulate the sampling method, which leads to the conclusion that a convenience sample was most likely used in these studies. Convenience sampling is prone to sampling bias and data from such samples are considered to be of an inferior level [71]. Full appreciation of the clinical value of the reported kinematic data also depends on the representativeness of the sample. Inclusion and exclusion criteria and the sample size (power) are important factors in this context. Approximately half of the manuscripts omitted to state clearly their inclusion and exclusion criteria and in about a third of the studies no more than 10 subjects were recruited [27,28,31,36,39,40,47,49]. Reporting the power, as performed by Long et al. [29], should therefore be an element of consideration in future studies. The quality of the demographic data was only adequate in half of the studies.

Full clinical validation of a gait related measurement tool is often pursued through an adequate repeatability study, including appropriate and, 'clinically relevant', statistical indices [20,21]. Studies reporting only the kinematic waveforms from one test session obtained from a small number of subjects can be considered, from a qualitative perspective, to represent the lowest level of repeatability. Studies considering the between-trial variability demonstrated that artefacts from skin movement at the foot occur in a systematic and consistent way and that they are of such a magnitude that they do not preclude the clinical utility in children [46,54], in healthy adults [37,39,41,55,64,67] and in impaired subjects $[43,66]$. A minority of studies investigated the between-day, between-trial, between-subject and between-clinician repeatability.

Most commonly, CMC and ICC values are reported as measure of waveform resemblances. However, evidence from the literature leads to the conclusion that such indices have only a limited significance and should therefore not be used in isolation $[21,72]$. In fact, it has been recommended to include measures of absolute measurement error (e.g. SD and SEM), in order to fully appreciate the reliability of the kinematic data. These absolute measurement errors are of critical importance in the decision making process, as it allows differentiation between 'true' and 'false' differences or effects. Considering the data from this study, it can be stated that such repeatability indices are available for only a minority of the foot models.

\section{Standardization}

Despite the remarkable progression made in the last two decades in the area of the kinematic analysis of the foot, it should be stressed that $3 \mathrm{D}$ multisegmental foot modelling, as defined above, lacks technical uniformity. A lack of uniformity was observed in the terminology describing the different foot segments. Full comparison of the motion patterns between models is often precluded due to non-uniformity in modelling of segments and a lack of a standard for reporting intersegmental motion. Development of an adequate reference position (zero position) method from which joint kinematics can be described has been the subject of much debate in the literature. The clinical definition commonly used for a zero position, being 'an anatomical pose from which a segment can move in both directions' [73], is probably too simplistic with regard to the complexity of the foot. The aforementioned lack of standardizations is also believed to conflict with the general opinion (goal) that 3DMFMs should be applicable to the assessment of both healthy and deformed feet $[41,55]$. It is believed that this opinion deserves full credibility and it can be considered as a logic consequence when international standardization is pursued. However, the complexity and the enormous variability in the clinical presentation of foot disorders may cause that axes for inter-segmental motion do not conform to the standard anatomical planes. Errors coming from such misalignment will have to be investigated and quantified in order to fully appreciate the validity of 3DMFMs in the presence of deformity.

Some consensus on terminology regarding shank, hindfoot, forefoot and hallux has been proposed some years ago by the attendees of the Special Technical Session of the Gait \& Clinical Movement Analysis Society (GCMAS) in 2003. Researchers should be encouraged to elaborate further on this topic in order to provide the international community with adequate guidelines. In this perspective, it was decided to describe the minimum factors that should be considered in the development and clinical validation of a 3DMFM (Additional file 3). Future direction for research should focus on optimization of current 3DMFMs. This includes the development of most 'optimal markers sets', the development of clinically applicable reference position(s) and the adoption of an optimal method for angular calculation. The development of disorder-specific models, as suggested by some authors [26], deserves further consideration.

\section{Conclusion}

Several 3DMFMs have been published and the clinical implementation of these seems to provide biomechanical parameters which can help clinicians in their decision making process. The systematic errors introduced by skin motion artifact and the difficulty of tracking specific bones in the foot seem to be manageable elements, not precluding an enhanced biomechanical insight. Investigations have demonstrated that a certain number of intersegmental angles can be measured in a consistent and repeatable way, even in the presence of foot pathologies. These findings have therefore provided evidence for the continued use of 3DMFMs.

However, in order to appreciate fully the clinical utility of the published foot models it is recommended that future studies focus on robust repeatability studies. Consideration should be given to between-day, between-trial, between-clinician and betweensubject repeatability (including patient populations). Moreover, not only correlation indices, but also absolute values of measurement error should be provided. These data will indicate the most robust inter-segmental parameters which can subsequently be used in the decision making process.

\section{Acknowledgments}

This review work was carried out under the financial support of the Agency for Innovation by Science and Technology Flanders (Grant: 080659). The work of G.A. Matricali was partially funded by a clinical doctoral scholarship of the Research Foundation-Flanders (Belgium). 
The authors also like to thank Dr. Ivan Birch for grammar and language editing.

\section{Conflict of interest statement}

The authors declare that no financial and personal relationships exist which could have influenced (bias) their work.

\section{Appendix A. Supplementary data}

Supplementary data associated with this article can be found, in the online version, at doi:10.1016/j.gaitpost.2010.12.018.

\section{References}

[1] Davis IS. How do we accurately measure foot motion? J Orthop Sports Phys Ther 2004;34:502-3.

[2] Davis IS. Foot ankle research retreat: consensus statement. J Orthop Sports Phys Ther 2004;34:A2-4.

[3] Westblad P, Hashimoto T, Winson I, Lundberg A, Arndt A. Differences in anklejoint anchored markers. Foot Ankle Int 2002;23(9):856-63.

[4] Arndt A, Wolf P, Lui A, Nester C, Stacoff A, Jones R, et al. Intrinsic foot kinematics measured in vivo during the stance phase of slow running. J Biomech 2007;40(12):2672-8.

[5] Robertson DG, Caldwell G, Hamill J, Kamen G, Whittlesey SM. Research methods in biomachanics. Leeds: Human Kinetics; 2004. pp. 9-52.

[6] Woodburn J, Nelson KM, Siegel KL, Kepple TM, Gerbber LH. Multisegment foot motion during gait: proof of concept in rheumatoid arthritis. J Rheumato 2004;31(10):1918-27.

[7] Baker R, Robb J. Foot models for clinical gait analysis. Gait Posture 2006;23: 399-400.

[8] Nester N, Jones RK, Lui A, Howard D, Lundberg A, Arndt A, et al. Foot kinematics during waking measured using bone and surface mounted markers. J Biomech 2007;40:3412-23.

[9] Nester CJ, Lui AM, Howard D, Cocheba J, Derrick T, Patterson P. In vivo study of foot kinematics using dynamic walking cadaver model. J Biomech 2007;40: 1927-37.

[10] Brown KM, Bursey DE, Arneson LJ, Andrews CA, Ludewig PM, Glasoe WM. Consideration of digitization precision when building local coordinate axes for a foot model. J Biomech 2009;42:1263-9.

[11] Okita N, Meyers SA, Challis JH, Sharkey NA. An objective evaluation of a segmented foot model. Gait Posture 2009;30:27-34.

[12] Apkarian J, Naumann S, Cairns B. A three-dimensional kinematic and dynamic model of the lower limb. J Biomech 1989;22(2):143.

[13] Kadaba MP, Ramakrishnan HK, Wootten ME. Measurement of lower extremity kinematics during level walking. J Orthop Res 1990;8(3):383-92.

[14] Davis RB, Ounpuu S, Tyrburski D, Gage JR. A gait analysis data collection and reduction technique. Hum Mov Sci 1991;10:575-87.

[15] Ounpuu S, Gage JR, Davis RB. Three-dimensional lower extremity joint kinetics in normal pediatric gait. J Pediatr Orthop 1991;11(2):341.

[16] Mulrow C, Coock DJ, Davidoff F. Systematic review: critical links to the great chain of evidence. Ann Int Med 1997;126:389-91.

[17] Higgins JPT, Green S, editors. Cochrane handbook for systematic review of interventions. Version 5.0.2 [update September 2009]. The Cochrane Collaboration; 2008. Available from: www.Cochrane-handbook.org.

[18] Capozzo A, Della Croce U, Leardini A, Chiari L. Human movement analysis using stereophotogrammetry. Part 1. Theoretical background. Gait Posture 2005;21:186-96.

[19] Skaggs DL, Rethlefsen SA, Kay RM, Dennis SW, Reynolds RAK, Tolo VT Variability in gait analysis interpretation. J Pediatr Orthop 2000;20:759-64.

[20] Schwartz MH, Trost JP, Wervey RA. Measurement and management of errors in quantitative gait data. Gait Posture 2004;20:196-203.

[21] McGinley JL, Baker R, Wolfe R, Morris M. The reliability of three-dimensional kinematic gait measurements: a systematic review. Gait Posture 2009;29(3): 360-9.

[22] Van Gheluwe B, Dananberg H, Hagman F, Vanstaen K. Effects of hallux limitus on plantar foot pressure and foot kinematics during walking. J Am Podiatr Med Assoc 2006;96(5):428-36.

[23] Chang R, Van Emmerik R, Hamill J. Quantifying rearfoot-forefoot coordination in human walking. J Biomech 2008;41:3101-5.

[24] Sawacha Z, Gabriella G, Cristoferi G, Guiotto A, Avogaro A, Cobelli C. Diabetic gait and posture abnormalities: a biomechanical investigation through three dimensional gait analysis. Clin Biomech 2009;24:722-8.

[25] Turner DE, Helliwel PS, Lohmann-Siegel K, Woodburn J. Biomechanics of the foot in rheumatoid arthritis: identifying abnormal function and the factors associated with localised disease' impact'. Clin Biomech 2007;23:93-100.

[26] Rankine L, Long J, Canseco K, Harris G. Multisegmental foot modelling: a review. Crit Rev Biomed Eng 2008;36:127-81.

[27] Kidder SM, Abuzzahab FS, Harris GF, Johnson JE. A system for the analysis of foot and ankle kinematics during gait. IEEE Trans Rehabil Eng 1996;4(1): 25-32.
[28] Myers KA, Wang M, Marks RM, Harris GF. Validation of a multisegment foot and ankle kinematic model for pediatric gait. IEEE Trans Rehabil Eng 2004;12(1):122-30.

[29] Long JT, Eastwood DC, Graf AR, Smith AP, Harris GF. Repeatability and sources of variability in multi-center assessment of segmental foot kinematics in normal adults. Gait Posture 2009;31(1):32-6.

[30] Khazzam M, Long JT, Marks RM, Harris GF. Preoperative gait characterization of patients with ankle arthrosis. Gait Posture 2006;24(1):85-93.

[31] Canseco K, Long J, Marks R, Khazzam M, Harris G. Quantitative characterization of gait kinematics in patients with hallux rigidus using the Milwaukee Foot Model. J Orthop Res 2008;26(4):419-27.

[32] Khazzam M, Long JT, Marks RM, Harris GF. Kinematic changes of the foot and ankle in patients with systematic rheumatoid arthritis and forefoot deformity. J Orthop Res 2007;25(3):319-29.

[33] Ness ME, Long J, Marks R, Harris G. Foot and ankle kinematics in patients with posterior tibial tendon dysfunction. Gait Posture 2007;27:331-9.

[34] Canseco K, Long J, Marks R, Khazzam M, Harris G. Quantitative motion analysis in patients with hallux rigidus before and after cheilectomy. J Orthop Res 2009;27(1):128-34.

[35] Marks RM, Long JT, Ness ME, Khazzam M, Harris GF. Surgical reconstruction of posterior tibial tendon dysfunction; prospective comparison of flexor digitorum longus substitution combined with lateral colum lengthening or medial displacement calcaneal osteotomy. Gait Posture 2008;29:17-22.

[36] Rattanaprasert U, Smith R, Sullivan M, Gilleard W. Three dimensional kinematics of the forefoot, rearfoot, and leg without the function of tibialis posterior in comparison with normal during stance phase of walking. Clin Biomech 1998;14:14-23.

[37] Cornwall MW, McPoil TG. Three dimensional movement of the foot during the stance phase of walking. J Am Podiatr Med Assoc 1999;89(2):56-66.

[38] Cornwall MW, McPoil TG. Motion of the calcaneus, naviculair and first metatarsal during the stance phase of walking. J Am Podiatr Med Assoc 2002;92(2):67-76.

[39] Leardini A, Benedetti MG, Catani F, Simoncini L, Giannini S. An anatomically based protocol for the description of foot segment kinematics during gait. Clin Biomech 1999;14:528-36.

40] Giacomozzi C, Benedetti MG, Leardini A, Marcellari V, Gianninni S. Gait analysis with an integrated system for functional assessment to talocalcaneol coalition. J Am Podiatr Med Assoc 2006;26(2):107-15.

41] Carson MC, Harrington ME, Thompson N, O'Conner JJ, Theologis TN. Kinematic analysis of a multi-segment foot model for research and clinical applications: a repeatability analysis. J Biomech 2001;34:1299-307.

[42] Theologis TN, Harrington ME, Thompson N, Benson MKD. Dynamic foot movement in children treated for congenital talipes equinovarus. J Bone Joint Surg 2003;85:572-7.

[43] Woodburn J, Nelson KM, Siegel KL, Kepple TM, Gerber LH. Multisegment foot motion during gait: proof of concept in rheumatoid arthritis. J Rheumatol 2004;31(10):1918-27.

[44] Turner DE, Helliwel PS, Emery P, Woodburn J. The impact of rheumatoid arthritis on foot function in the early stages of disease: a clinical case series. BMC Musculoskelet Disord 2006;7:102.

[45] Turner DE, Woordburn J. Characterising the clinical and biomechanical features of severely deformed feet in rheumatoid arthritis. Gait Posture 2008;28: 574-80.

[46] Stebbins J, Harrington M, Thompson N, Zavatsky A, Theologis T. Repeatability of a model for measuring multi-segment foot kinematics in children. Gait Posture 2006;23:401-10.

[47] Curtis DJ, Bencke J, Stebbins JA, Stansfield B. Intra-rater repeatability of the Oxford foot model in healthy children in different stages of the foot roll over process during gait. Gait Posture 2009;30:118-21.

[48] Alonso-Vazquez A, Villarroya MA, Franco MA, Asin J, Calvo B. Kinematic assessment of paediatric forefoot varus. Gait Posture 2008;29:214-9.

[49] Arampatzis A, Brüggemann G, Morey Klapsing G. A three-dimensional shankfoot model to determine the foot motion during landings. Med Sci Sports Exerc 2002;34(1):130-8.

[50] Arampatzis A, Morey Klapsing G, Brüggemann G. The effect of falling height on muscle activity and foot motion during landings. J Electromyogr Kinesiol 2003;13:533-44.

[51] Morey-Klapsing G, Arampatzis A, Brueggemann G. Joint stabilizing response to expected and unexpected tilts. Foot Ankle Int 2005;26(10):870-80.

[52] Morey-Klapsing G, Arampatzis A, Brüggemann G. Joint stabilizing response to lateral and medial tilts. Clin Biomech 2006;20:517-25.

[53] Morey-Klapsing G, Arampatzis A, Brüggemann G. Evidence of proactive forefoot control during landings on inclined surfaces. J Mot Behav 2007;39(2):89102.

[54] MacWilliams BA, Cowley M, Nicholson DE. Foot kinematics and kinetics during adolescent gait. Gait Posture 2003;17:214-24.

[55] Simon J, Doederlein L, McCintosh AS, Mataxiotis D, Block HG, Wolf SI. The Heidelberg foot measurement method: development, description and assessment. Gait Posture 2006;23:411-24.

[56] Wolf S, Simon J, Patikas D, Schuster W, Armbrust P, Doederlein L. Foot motion in children shoes - a comparison of barefoor walking with shod walking in conventional and flexible shoes. Gait Posture 2008;27: 51-9.

[57] Tome J, Nawoczenski D, Flemister A, Houck J. Comparison of foot kinematics between subjects with posterior tibialis tendon dysfunction and healthy controls. J Orthop Sports Phys Ther 2006;36(9):635-44. 
[58] Rao S, Baumhauer JF, Tome J, Nawoczenski DA. Comparison of in vivo segmental foot motion during walking and step decent in patients with midfoot arthritis and matched asymptomatic control subjects. J Biomech 2009;42:1054-60.

[59] Nawoczenski DA, Ketz J, Baumhauer JF. Dynamic kinematic and plantar pressure changes following cheilectomy for hallux rigidus: a mid-term follow up. Foot Ankle Int 2008;29(3):265-72.

[60] Leardini A, Benedetti MG, Berti L, Bettinelli D, Nativo R, Giannini S. Rear-foot mid-foot and fore-foot motion during the stance phase of gait. Gait Posture 2007;25:453-62.

[61] Rao S, Saltzman C, Yack HY. Segmental foot mobility in individuals with and without diabetes and neuropathy. Clin Biomech 2006;22:464-71.

[62] Houck JR, Tome JM, Nawozenski DA. Subtalar neutral position as an offset for kinematic model of the foot during walking. Gait Posture 2006;28:29-37.

[63] Houck J, Neville CG, Tome J, Flemister A. Foot kinematics during a bilateral heel rise test in participants with stage 2 posterior tibial tendon dysfunction. Orthop Sports Phys Ther 2009;39(8):593-603.

[64] Jenkyn TR, Nicol AC. A multi-segment kinematic model of the foot with a novel definition of forefoot motion for use in clinical gait analysis during walking. J Biomech 2007;40(14):3271-8.

[65] Jenkyn TR, Nicol AC. Foot segment kinematics during normal walking using a multisegment model of the foot and ankle complex. J Biomech Eng 2009;131:3.
[66] Sawacha Z, Cristoferi G, Guarneri G, Corazzo S, Dona G, Denti P, et al. Characterizing multisegment foot kinematics during gait in diabetic foot patients. J Neuroeng Rehab 2009;23(6):37.

[67] Cobb SC, Tis LL, Johnson JT, Wang YT, Geil MD, McCarty FA. The effect of lowmobile foot posture on multi-segment medial foot model gait kinematics. Gait Posture 2009;30:334-9.

[68] Cappozzo A, Catani F, Delle Croce U, Leardini A. Position and orientation in space of bones during movement: anatomical frame definition and determination. Clin Biomech 1995;10:171-8.

[69] Deville WL, Buntinx F, Bouter LM, Montori VM, De Vet HCW, Van der Windt DAW. Conducting systematic review of diagnostic studies: didactic guidelines. BMC Med Res Methodol 2002;3(2):9.

[70] Noonan KJ, Halliday S, Browne R, O’Brien S, Kayes K, Feinberg J. Interobserver variability of gait analysis in patients with cerebral palsy. J Pediatr Orthop 2003;23(3):279-87.

[71] Portney LG, Watkins MP. Foundations of clinical research application to practice, vol. 1. New Jersey: Prentice-Hall; 2000. p. 768.

[72] Yavuszer G, Oken O, Elhan A, Stam HJ. Repeatability of lower limb three dimensional kinematics in patients with stroke. Gait Posture 2008;27:31-5.

[73] Long JT, Wang M, Harris J, Gerald F. A multisegmental foot model with bonebased referencing: sensitivity to radiographic input parameters. Eng Med Biol Soc $2008 ; 879-82$. 